\title{
Entomología y control de vectores Enfermedad de Chagas
}

\section{Seguimiento de cuatro generaciones sucesivas de Triatoma infestans dark morph silvestre bajo condiciones controladas de laboratorio}

Agustín Balsalobre, Gerardo A. Martí, María L. Susevich, Julia Luna, Eliana Nieves, Yamila Obed, Jorge E. Rabinovich

Centro de Estudios Parasitológicos y de Vectores, (CEPAVE-CCT-La Plata-CONICET - UNLP), La Plata, Argentina

Introducción. Hasta el presente, son escasos los datos existentes en la bibliografía sobre parámetros de poblaciones silvestres de Triatoma infestans, e inexistentes, los de $T$. infestans dark morph, bajo condiciones controladas de laboratorio. Se compararon los parámetros de poblaciones de $T$. infestans dark morph a lo largo de cuatro generaciones sucesivas en laboratorio.

Materiales y métodos. Bajo condiciones contro-ladas de laboratorio $\left(28 \pm 1{ }^{\circ} \mathrm{C}, 40 \pm 5 \% \mathrm{HR}\right)$, se desarrolló una colonia de $T$. infestans a partir de huevos de una hembra dark morph fecundada y capturada en la Reserva del Loro Hablador, Chaco, Argentina. Se mantuvieron en lotes independientes los individuos de cada generación (F1 a F4). Se alimentaron semanalmente durante 45 minutos sobre gallina $y$ se registraron el número de huevos depositados y la mortalidad de cada estadio. A partir de la etapa adulta. el seguimiento fue individual y se colocaron cada semana machos y hembras en parejas al azar. Se analizaron las estadísticas vitales y los parámetros de población para todas las cohortes, mediante un programa estadístico en lenguaje Delphi.

Resultados. Hubo diferencias estadísticamente significativas desde la F1 a la F4, en el aumento en el tiempo de desarrollo de huevo a NV (de 23 a 33 semanas; $y=2.969 x+20,21, r=0,977$ ), y en la reducción en la tasa neta de reproducción (R0) (de $11,6$ a 0,$28 ; y=-4.012 x+15,58, r=0,980)$.

Conclusión. La forma dark morph de $T$. infestans no parece adaptarse a las condiciones del laboratorio. El número de hembras que reemplazaría en promedio a cada hembra en una generación (Ro), disminuye de manera sostenida con las sucesivas generaciones, llegando a ser menor que uno a la cuarta generación, y la población no podría reemplazarse a sí misma.

\section{Evaluación de Isaria fumosorosea y Metarhizium anisopliae para el control biológico de Meccus pallidipennis en condiciones de laboratorio}

Any Laura Flores-Villegas ${ }^{1}$, Margarita Cabrera-Bravo ${ }^{1}$, Armando Pérez-Torres ${ }^{3}$, Mauro Omar Vences-Blanco ${ }^{1}$, Hortensia Navarro-Barranco ${ }^{2}$, Miguel Ángel Ayala-

Zermeño², Irma López-Martínez ${ }^{3}$, Víctor Manuel

Hernández-Velázquez ${ }^{4}$ Paz María Salazar-Schettino", Conchita Toriello-Nájera ${ }^{2}$

1 Laboratorio de Biología de Parásitos

2 Laboratorio de Micología Básica, Departamento de Microbiología y Parasitología, Facultad de Medicina, Universidad Nacional Autónoma de México, México

3 Laboratorio de Inmunología Comparada de Piel y Mucosas, Departamento de Biología Celular y Tisular, Facultad de Medicina, Universidad Nacional Autónoma de México, México

4 Laboratorio de Control Biológico, Centro de Investigación en Biotecnología, Universidad Autónoma del Estado de Morelos, México

Introducción. Un componente clave del control integral de los transmisores de la enfermedad de Chagas, es el uso de insecticidas. Sin embargo, se han presentado problemas de resistencia, contaminación ambiental y riesgos para la salud humana, aunados a la problemática de control de los transmisores peridomésticos (Meccus pallidipennis). Los hongos entomopatógenos Isaria fumosorosea (EH/511-3) y Metarhizium anisopliae (EH/473-4) son capaces de controlar a diversos insectos que son plagas agrícolas.

El objetivo del estudio es evaluar, bajo condiciones de laboratorio, los cambios patológicos, el porcentaje de mortalidad y el tiempo letal medio (TLM) de M. pallidipennis, al ser expuesto a hongos entomopatógenos.

Materiales y métodos. Se determinó el TLM en 75 huevos de $M$. pallidipennis, tres bioensayos (25 huevos cada uno) con $2 \mu \mathrm{l}$ de una suspensión de $3 \times 10^{5}$ conidios $/ \mathrm{ml}$ de $I$. fumosorosea y $M$. anisopliae. Se incubaron a $28^{\circ} \mathrm{C}$ con fotoperiodo $12 \times 12$ h (luz-oscuridad). Se observaron 10 días y se registró la mortalidad cada 12 horas. Para comprobar los cambios patológicos, se utilizó microscopía electrónica de transmisión (MET).

Resultados. Se observó que la cepa EH/511-3 presentó el $92 \%$ de mortalidad a una concentración 
de $3 \times 10^{5}$ y el TLM fue de 66.685 horas (2,77 días). La cepa EH/473-4 mostró 89 \% de mortalidad y un TLM de 76.320 horas (3,18 días), a la misma concentración. La microscopía electrónica de transmisión demostró la presencia de propágulos fúngicos de ambos hongos dentro del embrión, destruyendo todas sus estructuras, y se observó la salida del hongo a través del vitelo hacia la superficie del huevo.

Conclusiones. I. fumosorosea mostró una elevada virulencia del hongo hacia los huevos de $M$. pallidipennis, lo cual sugiere que este entomopatógeno puede ser un candidato idóneo como agente microbiano para el control biológico del vector de la enfermedad de Chagas.

$$
\text { - } \bigcirc
$$

\section{Estudios genéticos de las especies del complejo Phyllosoma importantes vectores de la enfermedad de Chagas en el centro de México}

Bertha Espinoza', Fernando Martínez¹, Guiehdani Villalobos ${ }^{1}$, Alejandro Martínez ${ }^{2}$, Ricardo AlejandréAguilar $^{3}$, Silvia Catalá ${ }^{4}$, Patricia De la Torre ${ }^{1}$, Juan

Pedro Laclette ${ }^{1}$

1 Laboratorio de estudios sobre Tripanosomiasis Americana, Instituto de Investigaciones Biomédicas, Universidad Nacional Autónoma de México, México, D.F., México

2 Centro Universitario del Sur, Universidad de Guadalajara, Guadalajara, México

3 Escuela de Ciencias Biológicas del Instituto Politécnico Nacional, México

4 Centro Regional de Investigación Científica y Transferencia Tecnológica, La Rioja, Argentina

Introducción. La enfermedad de Chagas es una zoonosis causada por Trypanosoma cruzi, protozoario parásito. En la naturaleza, T. cruzi se mantiene principalmente en un ciclo selvático que involucra a diferentes especies de insectos triatominos (Hemiptera: Reduviidae) que actúan como vectores. Debido a diversos factores ambientales, México alberga numerosas especies endémicas, de las cuales, las pertenecientes al complejo Phyllosoma son de gran importancia por su distribución y adaptación al ambiente doméstico y peridoméstico. El objetivo del presente trabajo fue hacer un análisis genético que permitiera definir con precisión el estatus taxonómico de las especies que conforman el complejo Phyllosoma.

Materiales y métodos. Para el análisis genético de los vectores, se utilizó la secuenciación del espaciador transcrito interno 2 (ITS2) del ADN ribosómico y un fragmento del gene del citocromo
B (mtCYTB) del ADN mitocondrial, así como un estudio morfométrico antenal. Se estudiaron las siete especies que forman el complejo Phyllosoma $\mathrm{y}$, además, a $T$. dimidiata, $T$. rubida, $T$. lecticularia y Rhodnious prolixus. T. infestans se usó como grupo externo. Con los datos obtenidos se hizo un análisis genético y una reconstrucción filogenética.

Resultados. Los resultados genéticos mostraron que las siete especies del complejo Phyllosoma son muy próximas y que $T$. dimidiata es también muy cercana a este complejo. Por primera vez se demostró que $T$. lecticularia y $T$. rubida están también relacionados con el complejo Phyllosoma. Además, se describen individuos producto del cruce entre $T$. pallidipennis, $T$. picturata y $T$. longipennis, con características similares a los progenitores.

Conclusiones. El complejo Phyllosoma se encuentra constituido por subespecies o variedades con diferencias morfológicas y cromáticas que pueden entrecruzarse en la naturaleza.

Los resultados parciales de este trabajo se presentaron con anterioridad en otra reunión científica.

\section{Relação filogenética entre quatro espécies do subcomplexo rubrovaria baseada no sequenciamento parcial de citocromo B e citocromo oxidase 1}

Cláudia Solano Rocha1, Sueli Gardim¹, Marco Túlio

Alves da Silva², Carlos Eduardo Almeida', Regina Maria Barretto Cicarelli', João Aristeu da Rosa ${ }^{1}$

1 Faculdade de Ciências Farmacêuticas, UNESP, Araraquara, SP, Brasil

2 Instituto de Física, USP, São Carlos, SP, Brasil

Introdução. Atualmente estão descritas 144 espécies de Triatominae. Em estudos recentes, o gênero Triatoma foi agrupado em oito complexos e subcomplexos. Triatoma rubrovaria, $T$. circummaculata, T. klugie T. carcavalloisão espécies encontradas em ecótopos semelhantes no Estado do Rio Grande do Sul, Brasil, e foram agrupadas em um subcomplexo (subcomplexo Rubrovaria), baseadas em características morfológicas.

Objetivo. O presente estudo tem por objetivo inferir a relação filogenética entre as quatro espécies.

Material e métodos. A partir do DNA genômico de dois exemplares de cada espécie, foram amplificados e sequenciados fragmentos dos genes citocromo B (CytB) e citocromo oxidase I (COI). As sequências foram alinhadas e reunidas em um contig único, formando uma sequência de 1.069 pares de bases contendo os dois fragmentos. $T$. 
dimidiata foi utilizado como outgroup. A inferência Bayesiana foi realizada por meio do software MrBayes 3.1.2 seguindo o modelo AIC, obtido pelo software MrModeltest.

Resultados. O filograma obtido da análise dos dados combinados sugere T. klugi e T. carcavalloi como espécies irmãs (probabilidade Bayesiana de 99) e este clado, irmão ao $T$. circummaculata (probabilidade Bayesiana de 100) e ao de $T$. rubrovaria (probabilidade Bayesiana de 100), que juntos formam o "subcomplexo rubrovaria".

Conclusão. A análise filogenética corrobora a atual classificação baseada em características morfológicas das espécies em estudo, bem como a origem monofilética das mesmas.

Auxílio. FAPESP, processo 2009/52236-2.

\section{New discoveries of sylvatic Triatoma infestans throughout the Bolivian Chaco}

Etienne Waleckx, Stéphanie Depickère, Renata Salas, Claudia Aliaga, Marcelo Monje, Hiber Calle, Rosio Buitrago, François Noireau, Simone Frédérique Brenière MIVEGEC (Maladies Infectieuses et Vecteurs

: Ecologie, Génétique, Evolution et Contrôle),

Université Montpellier, Institut de Recherche pour le Développement, Montpellier, France et La Paz, Bolivia Instituto Nacional de Laboratorios de Salud (INLASA), Laboratorio de Entomología Médica, La Paz, Bolivia Escuela Técnica de Salud, Cochabamba, Bolivia IIBISMED, Facultad de Medicina, Universidad Mayor de San Simón, Cochabamba, Bolivia

Introduction. Triatoma infestans remains the most important and widespread vector of Trypanosoma cruzi, the causative agent of Chagas' disease. Sylvatic populations of the vector might be involved in the recolonization process of human dwellings. Nevertheless, knowledge about their distribution remains incomplete. To date, only one area with sylvatic T. infestans, discovered in 1997, has been reported in the Bolivian Chaco. The aim of this study was to improve our knowledge about the distribution and the ecology of sylvatic $T$. infestans populations in this region.

Materials and methods. An intensive search for triatomines was conducted using mouse-baited adhesive traps in 11 different sylvatic areas spread from north to south in the Bolivian Chaco. Caught triatomines weregenetically identified by sequencing of the nuclear rDNA ITS-2 region. Infection of the triatomines and genetic characterization of the parasites were investigated using the multiplex mini-exon PCR (MMPCR) method.
Results. Eighty-one sylvatic triatomines were caught. Thirty-eight were identified as $T$. infestans. Triatoma sordida and Panstrongylus geniculatus were the two other species collected. $T$. infestans and $T$. sordida specimens were all collected in hollow trees; $P$. geniculatus specimens were captured in burrows. One $T$. infestans and one $T$. sordida were infected with $T$. cruzi Tcl, and one $T$. infestans was infected with Tcll. The $T$. infestans specimens were captured in three areas further south (130-320 km) than the only area with sylvatic $T$. infestans previously recorded in the Bolivian Chaco. They were caught in sites either distant $(\approx$ $8 \mathrm{~km})$ or close $(<500 \mathrm{~m})$ to human dwellings.

Conclusion. These discoveries show that the epidemiological role of sylvatic $T$. infestans populations has to be considered carefully in the Bolivian Chaco. Moreover, they add to the current debate on the geographic distribution of sylvatic $T$. infestans populations and the geographic origin of the species.

\section{Comparación del ciclo de vida de Triatoma infestans mediante la alimentación en gallina y un alimentador artificial con sangre humana}

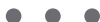

Gerardo A. Martí, Agustín Balsalobre, María L. Susevich, Jorge E. Rabinovich

Centro de Estudios Parasitológicos y de Vectores (CEPAVE-CCT-La Plata-CONICET - UNLP), La Plata, Argentina

Introducción. Desde la década de 1940 se han ensayado diferentes alimentadores artificiales adaptados especialmente para triatominos.

Se evaluaron los parámetros de la población de Triatoma infestans mediante la alimentación en gallina y un alimentador artificial con sangre humana.

Materiales y métodos. Dos cohortes de 45 ninfas de primer estadio, nacidas en un período de 48 horas, se mantuvieron en condiciones controladas de laboratorio. Ambas cohortes se alimentaron durante 45 minutos una vez por semana, una sobre gallina y otra con un alimentador artificial con sangre humana. Además, semanalmente se registró el número de huevos depositados y la mortalidad de los individuos por estadio y por sexos cuando adultos. Se calcularon las estadísticas vitales y los parámetros de población para ambas cohortes mediante un programa estadístico programado en lenguaje Delphi. 
Resultados. Para las cohortes en alimentador y en gallina se obtuvo, respectivamente: tiempo de desarrollo de huevo a NV, 28,5 y 18,3 semanas, tasa intrínseca de crecimiento natural $(r)$ de 0,02 y 0,03 , tasa finita de crecimiento de población $(\lambda)$ de 1,02 y 1,03 , tasa neta de reproducción $(R 0)$ de 11,82 y 20,14 , y tiempo generacional $(T)$ de 38,70 y 30,73 semanas. Además, en ambos casos se calcularon las estadísticas vitales (mortalidad y fecundidad específica por edades, y mortalidad pre-adulta específica por estadios).

Conclusiones. Los parámetros de población son alrededor de $65 \%$ mayores en alimentación sobre gallina que en alimentador artificial, el $\lambda$ alrededor de un $100 \%$ mayor, y el T alrededor de un $80 \%$ menor. Si el objetivo de los ensayos no son una producción masiva de insectos, el alimentador artificial es una excelente opción que permite trabajar con sangre en condiciones libre de gérmenes y, así, realizar experiencias o infecciones con diferentes parásitos y patógenos, como Trypanosoma, triatoma virus, Blastocrithidias, etc.

\section{Repelencia del aceite de citronela (Cymbopogon nardus) en refugios de adultos de Rhodnius prolixus (Reduviidae: Triatominae)}

Gisselle Rivera1', Helena Brochero², Jorge Molina'

1 Universidad de los Andes, Centro en Investigaciones en Microbiología y Parasitología Tropical, Bogotá, D.C., Colombia

2 Universidad Nacional de Colombia, Facultad de Agronomía, Bogotá, D.C., Colombia

Introducción. La presencia intradomiciliaria de Rhodnius prolixus es un factor de riesgo de infección de la enfermedad de Chagas. El desarrollo de estrategias de control vectorial con extractos naturales de plantas, ha demostrado ser eficaz y de poco impacto ambiental. El objetivo fue evaluar, en condiciones controladas de laboratorio, el efecto repelente del aceite de citronela en refugios de $R$. prolixus.

Materiales y métodos. El aceite de citronela se obtuvo por destilación con arrastre de vapor, extracción líquido-líquido y separación de las fases con un rotoevaporador. Las concentraciones evaluadas fueron puro, 1:10.000, 1:1.000, 1:10, $1: 5$ y 1:2. La arena experimental $\left(38.000 \mathrm{~cm}^{3}\right)$ recubierta con angeo se ubicó a $25^{\circ} \mathrm{C}$ (324.000 $\mathrm{cm}^{3}$ ) con luz blanca. Se evaluaron cuatro refugios en las esquinas de la arena (dos sin impregnar y dos impregnados) correspondientes a ladrillo o bahareque $\left(168 \mathrm{~cm}^{3}\right)$. Se evaluaron 20 triatominos adultos (10 machos y 10 hembras) para cada concentración del aceite y se emplearon 14 especímenes del mismo estadio como controles. Las preferencias se establecieron mediante conteos de los individuos en cada refugio a los 5 , $10,15,30$ y 60 minutos. Se utilizaron pruebas de Kruskall-Wallis para determinar el efecto del aceite de citronela sobre la escogencia.

Resultados. El sexo y tiempo de muestreo no mostraron diferencias significativas sobre la escogencia. Las diluciones 1:10.000, 1:1.000 o 1:10, no fueron eficaces en ninguno de los sustratos. Las diluciones 1:5 y 1:2 repelieron en el bahareque. El aceite sin diluir fue repelente para ambos sustratos.

Conclusión. Se encontró repelencia del aceite de citronela sobre $R$. prolixus dependiendo de la concentración y el sustrato. El aceite sin diluir puede considerarse como una opción para prevenir la transmisión vectorial de la enfermedad de Chagas.

\section{Hallazgo de vectores y reservorios en el brote de Chagas agudo de posible transmisión oral en Aguachica, Cesar}

Hugo Soto ${ }^{1}$, María Teresa Arias ${ }^{1}$, Marleny Montilla², Omar Triana ${ }^{3}$, Diana Carolina Suárez ${ }^{4}$, Ligia Lugo²,

Tania Tibaduiza ${ }^{2}$

1 Secretaría Departamental del Cesar, Valledupar, Colombia

2 Instituto Nacional de Salud, Bogotá, D.C., Colombia

${ }^{3}$ Universidad de Antioquia, Medellín, Colombia

${ }^{4}$ Universidad Industrial de Santander, Bucaramanga, Colombia

Introducción. En junio de 2010 se notificaron cinco casos probables de enfermedad aguda de Chagas procedentes del municipio de Aguachica, Cesar. Las personas se relacionaban en espacio, lugar y tiempo. El objetivo de este estudio fue identificar posibles vectores y reservorios implicados en la transmisión de Trypanosoma cruzi, en el brote de enfermedad aguda de Chagas por posible transmisión oral, de Aguachica, Cesar.

Materiales y métodos. Es un estudio descriptivo y transversal. Se hicieron reconocimiento geográfico, identificación de factores de riesgo de 58 viviendas, búsqueda de vectores en intradomicilio, peridomicilio y extradomicilio, estudio parasitológico y molecular de triatominos y estudio de reservorios, y aislamiento del parásito de paciente del líquido pericárdico a las cuatro semanas en medio de cultivo NNN y medio LIT con SBF al $10 \%$ y del triatomino $R$. prolixus pasándola por ratón y a las 
cuatro semanas sembrada en medio de cultivo Tobie y LIT.

Resultados. No se evidenció domiciliación de triatominos. Se recolectaron 24 ninfas de triatominos en una palma de ñoli (Elaeis oleífera), a 10 metros de la vivienda de los casos. La especie predominante fue Rhodnius pallescens (95,9\%). Otras especies recolectadas fueron Panstrongylus geniculatus y Eratyrus cuspidatus. El índice de infección con $T$. cruzi fue de $54,2 \%$. Se capturó un reservorio Didelphys marsupialis. El 58,6\% de los habitantes reconocían los triatominos, el 22,4\% dijeron verlos fuera de las viviendas y sólo el 5,2\% dijeron haberlos visto dentro de la vivienda. El 74,1 $\%$ de viviendas eran casas de ladrillo, de estas sólo el $29 \%$ se encontraban totalmente pañetadas, el $77,6 \%$ de las viviendas tenían piso de cemento, y el $91,4 \%$ tenían tejas de cinc. Se evidenció que $44,7 \%$ de las viviendas tenían construcciones unidas o cercanas a la casa principal y, en $36,1 \%$ de ástas, dormían animales domésticos.

Se examinaron 49 palmas de vino (Attalea butyracea) del casco urbano del municipio de Aguachica, se recolectaron $103 R$. pallescens, índice de infestación 28,6 \% e índice de infección de $70,9 \%$. Los análisis moleculares confirmaron la infección natural con $T$. cruzi l en triatominos de la especie $R$. pallescens recolectados en palmas.

Conclusiones. Los hallazgos demuestran la presencia de colonias grandes de $R$. pallescens en palmas (Attalea butyracea) del casco urbano del municipio de Aguachica, los cuales pueden ser fuente para la propagación de vectores infectados con $T$. cruzi I y, de esta manera, contribuir con el ciclo de transmisión para la enfermedad de Chagas.

$$
\text { - }-
$$

\section{The effect of simultaneous infection with Trypanosoma cruzi and Trypanosoma rangeli on Rhodnius prolixus survival}

Jennifer K. Peterson

Department of Ecology and Evolutionary Biology, Princeton University, Princeton, New Jersey, USA Grupo Biología y Control de Enfermedades Infecciosas, Sede de Investigación Universitaria, Medellín, Colombia

Introduction. Trypanosoma cruzi in human populations has been well studied but less understoodistheecology ofitssympatriccoexistence alongside $T$. rangeli within triatomines.

The objective of this study was to understand if Rhodnius prolixus individuals infected with both $T$. cruziand $T$. rangeli have different survival rates than those infected with just one of the trypanosomes.

Materials and methods. 71 R. prolixus fifth instar nymphs were allowed 30 minutes to feed on human blood infected with epimastigotes of $T$. cruzi, $T$. rangeli, $T$. cruzi and $T$. rangeli, or no parasites as a control. Blood was prepared by replacing the human serum with fetal bovine serum and adding parasites at an estimated concentration of 4 million per milliliter of blood. Insects monitored for 7 weeks post-infection and survival was recorded daily. Insect feces and hemolymph were examined weekly for parasites and infections were confirmed through PCR using primers specific for $T$. cruzi or T. rangeli.

Results. Individuals co-infected with both $T$. cruzi and $T$. rangeli died at significantly higher rates between 10 and 18 days post-infection. This time period coincides with when triatomines become able to transmit $T$. cruzi. However, after 18 days, insect death rates became significantly correlated with sheer number of parasites initially consumed rather than parasite species. We hypothesize that the temporary increase in death rates between 10 and 18 days is due to the simultaneous nature of the infection of both parasites and experiments are currently under way to test this hypothesis.

\section{Primer registro de Trypanosoma por biología molecular en Triatoma nigromaculata en el Cauca, Colombia}

Lina M. Villamil', Gustavo A. Vallejo'1, Noralba Montaño², Martha I. Ordóñez ${ }^{2}$, Deisy Y. Ahumada², Luz Eidi

Trujillo², Eryc Muñoz ${ }^{3}$, Luis Reinel Vásquez ${ }^{2}$

1 Laboratorio de Investigación en Parasitología Tropical, Programa de Biología, Universidad del Tolima, Ibagué, Colombia

2 Centro de Estudios en Microbiología y Parasitología, Programa de Enfermería y Departamento de Medicina Interna, Facultad Ciencias de la Salud, Universidad del Cauca, Popayán, Colombia

3 Hospital de El Tambo, Cauca, Colombia

Introducción. En el departamento del Cauca, Colombia, se conoce de hallazgos de vectores de Trypanosoma cruzi, tales como Triatoma nigromaculata, en El Tambo, Panstrongylus geniculatus, en Isla Gorgona y Piamonte (dato no publicado), y $P$. rufotuberculatus, en Santander de Quilichao. Sin embargo, no se ha descrito la presencia del parásito en estos vectores ni en humanos.

Se describe por primera vez para el Cauca y Colombia, el hallazgo por biología molecular de $T$. 
cruzi en $T$. nigormaculata procedentes de la vereda San Joaquín, corregimiento Playa Rica, El Tambo. Materiales y métodos. Se capturaron tres vectores, $T$. nigromaculata, en la vereda San Joaquín, corregimiento Playa Rica del municipio de El Tambo, Cauca. El grupo de investigación del Laboratorio de Investigación en Parasitología Tropical realizó una reacción de PCR dúplex, con los primers S35/S36/KP1L, que permiten la identificación de $T$. cruzi o $T$. rangeli. Se intentó identificar el genotipo de $T$. cruzi $(T$. cruzi I o $T$. cruzi II) utilizando los primers TCC/TC1/TC, pero no se obtuvo amplificación, debido probablemente a que esta reacción de PCR es menos sensible y requiere mayor cantidad de ADN.

Resultados. Se evidenció la presencia de T. cruzi sensu lato en dos de los vectores y una infección mixta de $T$. cruzi y $T$. rangeli $\mathrm{KP} 1(+)$ en uno de los vectores. No fue posible identificar el genotipo de $T$. cruzi debido a la poca cantidad de ADN.

Conclusiones. Se registra por primera vez para Colombia el hallazgo de $T$. cruzi y $T$. rangeli en $T$. nigromaculata procedente del departamento del Cauca. Es necesario ampliar el estudio en esta zona del país.

\section{Estudio de la distribución espacial de triatominos en el estado de Oaxaca, México}

Luis A. Hernández-Osorio ${ }^{1,4}$, Ricardo Alejandre-Aguilar², Josefina C. Trujillo-García ${ }^{3}$, Rebeca G. Manning-Cela ${ }^{1}$

1 Departamento de Biomedicina Molecular, CINVESTAV-IPN, México D.F., México

2 Departamento de Parasitología, Escuela Nacional de Ciencias Biológicas-IPN

${ }^{3}$ Laboratorio Estatal de Salud Pública de Oaxaca, México

${ }^{4}$ Facultad de Ciencias Químicas, Universidad Autónoma Benito Juárez de Oaxaca, México

Se reconocen 118 especies de triatominos, agrupados en 14 géneros y 5 tribus. En México se han identificado siete géneros, entre los que destaca el género Triatoma, con 21 especies distribuidas en todo el país. De acuerdo con su hábitat, se dividen en especies silvestres, domésticas y peridomésticas. Oaxaca tiene un amplio abanico de condiciones fisiográficas y climáticas, y se han reportado nueve especies de triatominos.

Con la finalidad de conocer la distribución de las especies más importantes, se hizo un análisis de triatominos recolectados (472) en áreas domésticas y peridomésticas de Oaxaca.

Utilizando el software MapInfo 10, se construyeron los mapas de distribución espacial por especie, donde se encontró que Triatoma barberi, $T$. mazzotti, $T$. dimidiata y $T$. phyllosoma son las especies más frecuentes. En zonas por encima de 1.000 metros sobre el nivel de mar (msnm), se encuentra principalmente $T$. barberi, compartiendo algunas regiones con $T$. mazzotti, $T$. dimidiata y T. phyllosoma. Triatoma mazzotti se encuentra ampliamente distribuida en la costa oaxaqueña, en regiones cálidas y cercanas al nivel del mar, y comparte amplias regiones con $T$. dimidiata y $T$. phyllosoma. Laespecie principal en la región del lstmo de Tehuantepec, fue T. phyyllosoma, que también se encuentra a lo largo de la costa compartiendo regiones con $T$. dimidiata y $T$. mazzotti.

Finalmente, $T$. dimidiata, aunque se encuentra predominantemente en zonas cálidas, también se encontró en zonas de la sierra sur por encima de $1.000 \mathrm{msnm}$. Las especies T. barberi, T. dimidiata y T. phyllosomapresentaron un porcentaje de infección entre 94 y $100 \%$, mientras que T. mazzotti mostró uno de 44 a $80 \%$. En algunos casos se encontró que, en el intradomicilio, cohabitaban T. phyllosoma con T. mazzotti en el istmo de Tehuantepec, $T$. mazzotti con $T$. dimidiata en la costa, y $T$. barberi con T. pallidipennis en la mixteca.

\section{Estandarización de las condiciones de ayuno y peso en ninfas de $V$ instar de Panstrongylus geniculatus para su utilización en pruebas biológicas}

Lyda Esteban, Víctor Manuel Angulo

Centro de Investigaciones en Enfermedades Tropicales, Universidad Industrial de Santander, Bucaramanga, Colombia

Introducción. Panstrongylus geniculatus es una especie que ha aumentado su importancia en salud publica en el país, debido a que recientemente se ha asociado a casos de enfermedad oral de Chagas. Debido a que no existen estudios acerca de la sensibilidad a insecticidas, urgen estudios en los cuales la primera etapa sea la obtención de gran cantidad de insectos para la realización de los bioensayos, la estandarización del tiempo de ayuno y el peso que deben establecerse para cada especie.

Materiales y métodos. Se utilizó una cepa de $P$. geniculatus colonizada en el año 2003 y mantenida en condiciones de laboratorio, para establecer la homogeneidad del peso antes y después de la ingestión, en ninfas de $\mathrm{V}$ instar. Una vez establecido el efecto de la ingestión sanguínea, se tomaron 
insectos sin alimentar a los 4, 5, 6, 7, 9, 11 y 14 días, y se pesaron. Se calcularon los rangos de las frecuencias de los pesos y el coeficiente de variación, y se levó a cabo la estadística descriptiva.

Resultados. El coeficiente de variación (Cv) demostró que el peso de las ninfas es más homogéneo en condiciones de ayuno, sin tener en cuenta el número de días de mudadas; varía así: Cv de 13,44 a 18,97 antes de la ingestión y de 25,64 a 44,38 después de la ingestión. El rango de pesos más homogéneo está comprendido entre los 50 y $80 \mathrm{mg}$ a los cinco días de ayuno $(79,4 \%)$ y entre 40 y $70 \mathrm{mg}$ a los nueves días de ayuno (85\%).

Conclusión. El ayuno favoreció la homogeneidad del peso de las ninfas. A los nueve y cinco días de ayuno, se logró un alto porcentaje de ninfas con pesos homogéneos. Sin embargo, se sugiere utilizarlas a los cinco días, pues esto podría disminuir el riesgo de muerte por ayuno prolongado en la lectura de los ensayos después de las pruebas toxicológicas.

Financiación. Colciencias 110249326216 y Secretaría de Salud de Santander.

\section{Estudio comparativo de la ovipostura de una cepa de laboratorio y una de campo de Panstrongylus geniculatus alimentadas con Gallus domesticus y Mus musculus BALB/c en condiciones de laboratorio}

Lyda Esteban, Víctor Manuel Angulo

Centro de Investigaciones en Enfermedades Tropicales, Universidad Industrial de Santander, Bucaramanga, Colombia

Introducción. Con el propósito de optimizar la ovipostura de las hembras de Panstrongylus geniculatus para la cría masiva de esta especie en condiciones de laboratorio y su utilización en bioensayos toxicológicos. Se compararon dos cepas, una de campo y una de laboratorio, a las cuales se ofrecieron dos fuentes sanguíneas.

Materiales y métodos. Se evaluaron dos cohortes de la cepa de laboratorio y una de la cepa de campo; cada cohorte estuvo conformada por diez hembras y diez machos individualizados. Adultos de la F1 constituyeron la cepa de campo, la cual se colonizó a partir de dos hembras y dos machos recolectados en zona rural del departamento de Santander, en 2010. La cepa de laboratorio consistió en adultos con un tiempo de colonización de ocho años. La alimentación fue semanal, al igual que el registro de la fecundidad, esto durante cuatro semanas.
Resultados. La fecundidad de la cepa de laboratorio alimentada con Gallus domesticus fue 687 y 619 huevos, con un promedio de 2,17 huevos por hembra y por día, mientras que en la cepa de campo fue 327 con 1,09 huevos por hembra y por día. La alimentación con ratón albino produjo aproximadamente 1,20 veces más huevos en la cepa de laboratorio, mientras que en la cepa de campo fue de 0,75 veces.

Conclusión. Las hembras de la cepa de laboratorio producen mayor número de huevos por mes, en comparación con la cepa de campo. La alimentación con ratón incrementó la fecundidad de $P$. geniculatus, más notable en las cepas de laboratorio que en la de campo.

Financiación. Colciencias 110249326216 y Secretaría de Salud de Santander.

\section{Relaciones filogenéticas entre varias especies de triatominos vectores de Trypanosoma cruzi en México con base en el ITS-2 del ADN ribosómico nuclear}

M. A. Zuriaga'1, M. L. Galván-Ramírez², S. Mas-Coma', M. D. Bargues ${ }^{1}$

1 Departamento de Parasitología, Facultad de Farmacia, Universidad de Valencia, Burjassot, Valencia, España

2 Laboratorio de Neurofisiología, Centro Universitario de Ciencias de la Salud, Universidad de Guadalajara, Sierra Mojada 950, Col. Independencia, Guadalajara, Jalisco, México

Introducción. La enfermedad de Chagas constituye un importante problema de salud pública en México, donde el control de la misma se complica por la existencia de un gran número de especies de triatominos vectores. Existen 14 especies de importancia epidemiológica en la transmisión de la enfermedad de Chagas en México, entre las que destacan las especies del complejo phyllosoma ( $T$. phyllosoma, T. longipennis, T. picturata, T. mazzottii, $T$. pallidipennis y T. bassolsae) en la costa oeste del país, y $T$. mexicana y $T$. gerstaeckeri en la costa este y los estados centrales, respectivamente.

Material y métodos. El presente estudio utiliza el segundo espaciador interno transcrito del ADN ribosómico nuclear (ITS-2), ampliamente utilizado y de gran utilidad en triatominos, para determinar los parentescos evolutivos de estas especies y tratar de resolver sus problemas a nivel taxonómico. El ADN se extrajo por métodos estandarizados, y el ITS-2 fue amplificado por PCR y secuenciado por el método de Sanger. 
Resultados y conclusiones. Los análisis de las secuencias y de los distintos haplotipos obtenidos, así como las relaciones filogenéticas inferidas con base en el ITS-2, nos indican que no se pueden establecer límites genéticos claros entre T. phyllosoma, T. longipennis, T. picturata, T. mazzottii, T. pallidipennis y $T$ bassolsae, por lo que se muestran como taxones muy próximos entre sí, para los cuales el rango de subespecie sería el más apropiado. El ITS-2 no permite incluir a T. mexicana y T. gerstaeckeri, en este complejo, aunque por sus características morfológicas y distribución geográfica, se planteó su inclusión en el mismo.

Estudio financiado por los Proyectos № ISCIII2005PI050574 y № ISCIII-RETIC RD06/0021/0017 de la Red de Investigación de Centros de Enfermedades Tropicales del FIS, Ministerio de Sanidad, España. El material utilizado en este estudio fue proporcionado por los miembros de la red ECLAT.

$$
\bullet \bullet
$$

\section{Sensibilidad al principio activo deltametrina en ninfas de primero y quinto estadios de Panstrongylus geniculatus}

Marlene Reyes, Lyda Esteban, Víctor Manuel Angulo Centro de Investigaciones en Enfermedades Tropicales, Universidad Industrial de Santander, Bucaramanga, Colombia

Introducción. Los piretroides son insecticidas utilizados para el control de triatominos domiciliados. Sin embargo, la intrusión de triatominos silvestres a las viviendas urbanas y rurales, requiere la evaluación de las estrategias actuales de control. Por tal motivo, se evaluó la sensibilidad de Panstrongylus geniculatus al principio activo deltametrina en ninfas de primer y quinto estadios. Metodología. Se aplicó el protocolo de evaluación de efecto insecticida sobre triatominos OMS, 1994, adaptado para esta especie, mediante la técnica de aplicación tópica. A partir de una cepa de $P$. geniculatus mantenida en condiciones constantes desde el 2003, se obtuvieron ninfas de primer estadio con 24 a 36 horas después de la eclosión y con un peso de 0,85 $\pm 0,13 \mathrm{mg}$, y ninfas de quinto estadio con cinco días de ayuno después de la muda y con un peso de 110,62 $\pm 19,24 \mathrm{mg}$. Se calcularon los parámetros estadísticos DL50 y DL99 usando el Probit and logit Analysis, versión 2.0, POLO PLUS.

Resultados. En ninfas de primer estadio, la DL fue $50 \mathrm{ng} /$ insecto=0,107 $\left(\mathrm{IC}_{95 \%} 0,080-0,0143\right)$ y la
$\mathrm{DL}_{99}$ fue 4,909 (IC $\left.\mathrm{I}_{95 \%} 2,154-19,192\right)$; en las ninfas de quinto estadio, la $\mathrm{DL}$ fue $50 \mathrm{ng} / \mathrm{insecto}=9.900$ $\left(\mathrm{IC}_{95 \%} 8,439-11,385\right)$ y la $\mathrm{DL}_{99} 66.513\left(\mathrm{IC}_{95 \%} 44,007-\right.$ 137,087).

Conclusiones. La estandarización de la línea base sensible en $P$. geniculatus (cepa UIS) a los insecticidas usados en los programas de control (deltametrina), permite contar con una herramienta que, con parámetros toxicológicos, permita iniciar los estudios de seguimiento de la resistencia en poblaciones de $P$. geniculatus.

Financiación. Colciencias COD: 110249326216 y Secretaría de Salud de Santander.

\section{Ensayos para mejorar la fecundidad de Eratyrus mucronatus en condiciones de laboratorio}

Mónica Flórez, Víctor Manuel Angulo

Centro de Investigaciones en Enfermedades Tropicales, Universidad Industrial de Santander, Bucaramanga,

Colombia

Introducción. Eratyrus mucronatus es un vector secundario de la enfermedad de Chagas, con reportes de domiciliación en algunas zonas de Colombia y Venezuela. Los pocos estudios realizados muestran que, en condiciones de laboratorio, tiene bajos porcentajes de eclosión y altas tasas de mortalidad en su ciclo de vida. El fin de este trabajo fue establecer condiciones que mejoren la fecundidad de esta especie para su cría en laboratorio.

Materiales y métodos. Sesenta hembras y 60 machos de E. mucronatus, de 2 a 3 meses denacidos, fueron puestos en parejas durante siete días, en un frasco plástico con los siguientes soportes (10 parejas por soporte): 1) cartón cartulina; 2) cartulina negra y cartón cartulina; 3) cartulina negra; 4) cartulina negra y hojas de palma; 5) cartulina negra y plumas de gallina; y 6) cartulina negra y cuero de cabra. El experimento se repitió seis veces por cada soporte. Al inicio de cada tratamiento, las hembras se pesaron antes y después de ofrecerles sangre de gallina. Para la comparación entre soportes, sólo se tuvieron en cuenta los huevos puestos por hembras alimentadas y el nivel de significancia se consideró con un $\mathrm{IC}_{90 \%}$.

Resultados. Se obtuvieron 4.467 huevos de $E$. mucronatus; 60 hembras semanalmente pueden poner 744,5 $\pm 120,77$ huevos; el promedio de oviposición diario por hembra estuvo en el rango de 1,19 a 2,69. Sólo se observaron diferencias significativas $(p=0,0787)$ en la fecundidad entre 
los soportes uno y cinco. Las hembras que no ingirieron sangre, no pusieron huevos.

Conclusiones. La producción de huevos de $E$. mucronatus en condiciones de laboratorio, puede ser mejorada usando plumas de gallina. Sin embargo, una alimentación semanal en pequeños grupos de individuos que asegure una alimentación completa, garantiza una mejora en las tasas de fecundidad de esta especie.

Financiación. Colciencias 110249326216 y Secretaría de Salud de Santander.

$$
\text { - }
$$

\section{Condiciones de cría y uso de ninfas $\mathrm{V}$ de Rhodnius pallescens para ensayos biológicos}

Mónica Flórez, Víctor Manuel Angulo

Centro de Investigaciones en Enfermedades Tropicales, Universidad Industrial de Santander, Bucaramanga, Colombia

Introducción. Rhodnius pallescens es una especie silvestre que ha tomado importancia como vector de la enfermedad de Chagas, por hacer intrusión en zonas sin poblaciones domiciliadas de Triatominae, en las cuales se han presentado brotes agudos de la enfermedad de Chagas en Santander. Se quiso establecer condiciones de cría de ninfas que permitan su mejor aprovechamiento en ensayos biológicos realizados para detectar y supervisar la resistencia a insecticidas.

Materiales y métodos. Teniendo en cuenta el protocolo de la OMS y el desarrollado en el CINTROP-UIS por Reyes y Angulo (2009), para el uso de ninfas de $V$ estadio en ensayos toxicológicos, se utilizó una cepa de laboratorio de $R$. pallescens (San Martín, Cesar) alimentada con gallina y se comparó el porcentaje de ninfas obtenido con los tratamientos: 1) ninfas- $V$ alimentadas a los 7 días de ayuno y pesadas a los 7 días; 2) ninfas-V alimentadas desde ninfa-I cada 8 días, y pesadas a los 5 y 6 días de ayuno; 3) Y ninfas- $V$ alimentadas desde ninfa-I cada 15 días y pesadas a los 5 y 6 días de ayuno. También se evaluó el tiempo de ofrecimiento de alimento.

Resultados. Con el primer tratamiento (100 a 200 $\mathrm{mg}$ ), se aprovecharían $71 \%$ de las ninfas-V; con el segundo (30 a $50 \mathrm{mg}$ ), $50 \%$ a los 5 días y $80 \%$ a los 6; y con el tercero (20 a 40 mg) $88 \%$ a los 5 días y $89 \%$ a los 6 días. Tiempo de alimentación: individuos que no comen a los 30 minutos, $40 \%$, a los 40 minutos, $18 \%$, a los 50 minutos, $13 \%$, y a los 60 minutos, $5 \%$.

Conclusiones. La cría de $R$. pallescens en las condiciones del tercer tratamiento y con un tiempo de alimentación de por lo menos 50 minutos, ofrece el menor esfuerzo y mejor aprovechamiento de ninfas- $V$ para realizar bioensayos cuando se requiere una gran cantidad de ninfas.

Financiación. Colciencias 110249326216 y Secretaría de Salud de Santander.

$$
\text { - } \bullet
$$

\section{Fuentes alimentarias de triatominos} (Hemiptera, Reduviidae, Triatominae) intrusos capturados por vigilancia comunitaria en Santander, Colombia

Mónica Flórez, Yeny Zulay Castellanos, Víctor Manuel Angulo, Ana Elvira Farfán

Centro de Investigaciones en Enfermedades Tropicales, Universidad Industrial de Santander, Bucaramanga, Colombia

Introducción. Losinsectoshematófagos dehábitats silvestres, como la mayoría de las especies de triatominos, cada día son más frecuentes visitantes de las viviendas rurales y urbanas. La identificación de sus fuentes alimentarias permite conocer sus huéspedes e indagar sobre su comportamiento y riesgo para transmitir Trypanosoma cruzi. La vigilancia comunitaria es el método más efectivo para supervisar la presencia de estos insectos en las viviendas.

Materiales y métodos. Se utilizaron triatominos que ingresaron al laboratorio de entomología del Centro de Investigaciones en Enfermedades Tropicales, durante los años 2009 y 2010, recolectados mediante la vigilancia entomológica llevada a cabo por la comunidad en varios municipios del departamento de Santander. Cada ejemplar fue determinado de acuerdo con la clave de Lent y Wygodzinsky (1979) y, para establecer sus fuentes alimentarias, se extrajo su contenido intestinal y se procesó con la técnica ELISA estandarizada por Farfán (2007), utilizando 18 antisueros policlonales específicos anti-especie animal.

Resultados. Se procesó el contenido intestinal de 11 triatominos de tres especies, provenientes de viviendas de cinco veredas y tres zonas urbanas de seis municipios de Santander. Se determinó la reactividad a proteínas sanguíneas de cinco antisueros, así: uno de dos ejemplares de Triatoma venosa a quiróptero, uno de cinco Rhodnius pallescens a dasipódido y un ejemplar de Panstrongylus geniculatus a didélfido; y otro de cuatro de esta misma especie a una mezcla de bovino y felino.

Conclusiones. La detección de sangre de animales silvestres en estas especies de triatominos confirma 
sus hábitos silvestres. La identificación de sangre de bovino, didélfido y felino en $P$. geniculatus muestra su carácter ecléctico y sinantrópico, y se destaca $T$. venosa, cuyos huéspedes y hábitos eran desconocidos. Se contribuye al conocimiento y comprensión del comportamiento de especies intrusas en zonas urbanas y rurales de Santander, cuya recolección fue posible gracias a la vigilancia comunitaria.

Financiación. Colciencias 110249326216 y Secretaría de Salud de Santander.

\section{Comportamiento de Rhodnius prolixus (Stal, 1859) frente a trampas cebadas con productores de $\mathrm{CO}_{2}$ en condiciones de laboratorio}

Ricardo González, Laurie Bruzual, Renny Alzola, Ana Ruotolo

Laboratorio de Vectores de Enfermedades Tropicales, Universidad de Oriente, Núcleo Bolívar, Venezuela

Introducción. La enfermedad de Chagas y sus transmisores guardan una estrecha relación con los factores sociales y ecológicos, fundamentalmente en áreas rurales. Rhodnius prolixus es el vector más importante en Venezuela. El estudio de su comportamiento es de gran importancia en su epidemiología.

Objetivo. La presente investigación evalúa el comportamiento de $R$. prolixus frente a trampas cebadas con productos liberadores de $\mathrm{CO}_{2}$ : cultivo de levadura e hielo seco.

Materiales y métodos. Se utilizaron ninfas de $R$. prolixus mantenidas en el laboratorio. Los ensayos se realizaron en un recipiente experimental de vidrio de $100 \times 100 \mathrm{~cm}$ de longitud por lado, por $20 \mathrm{~cm}$ de alto, con un piso de arena, cubierto por papel. Se realizaron tres experimentos. En el primero se utilizaron trampas cebadas con cultivo de levadura. Otras trampas contenían hielo seco. En ambos casos se usaron trampas control con solución de sacarosa. En un último experimento se usaron trampas cebadas con solución de levadura enfrentadas a trampas con hielo seco. Las experiencias se hicieron con y sin refugio para los insectos.

Resultados. Las trampas cebadas con cultivo de levadura atrajeron un número significativamente mayor de $R$. prolixus donde no había refugio. Del total de insectos capturados o atraídos en ambas situaciones experimentales, se observaron diferencias estadísticamente significativas en la atracción de los triatominos con el cultivo de levadura. Con el hielo seco no se observaron diferencias estadísticamente significativas. Cuando se enfrentaron el cultivo de levadura y el hielo seco, los resultados mostraron que las trampas cebadas con cultivo de levadura atrajeron un número mayor de insectos.

Discusión. Se demuestra que las trampas cebadas con el cultivo de levadura, y en menor grado el hielo seco, se comportan como una buena atracción para $R$. prolixus, lo cual coincide con los resultados de otros trabajos con otras especies de triatominos.

\section{Evidencias de la domiciliación de Eratyrus mucronatus (Reduviidae: Triatominae) infectado naturalmente con Trypanosoma cruzi, Cúcuta, Colombia}

Rocío Cárdenas ${ }^{1}$, Reinaldo Gutiérrez2, Claudia Magaly Sandoval ${ }^{1}$, Omaira Ortiz ${ }^{3}$, Andrea Portillo ${ }^{3}$, Leidy

Angarita ${ }^{3}$, Eulides Pabón ${ }^{4}$

1 Instituto Experimental "JWT" ULA, NURR, Venezuela

2 INBIOM, Universidad de Pamplona, Colombia

3 Programa de Bacteriología, UDES, Cúcuta, Colombia

${ }^{4}$ Instituto Departamental de Salud, Norte de

Santander, Colombia

Introducción. Eratyrus mucronatus (Stål, 1859) (Reduviidae: Triatominae) es considerado un eslabón activo del ciclo enzoótico de Trypanosoma cruzi, agente etiológico de la enfermedad de Chagas, que ha mostrado tendencias sinantrópicas en Colombia, Venezuela y Bolivia. En la ciudad de Cúcuta, principal zona de la frontera colombovenezolana, se detectó la presencia de $E$. mucronatus en un área urbana, por parte de funcionarios del grupo control de vectores del Instituto Departamental de Salud de Norte de Santander.

Metodología. Se visitaron y caracterizaron las viviendas del barrio Cuberos Niño $\left(7^{\circ} 52^{\prime} 16,8^{\prime \prime} \mathrm{N}\right.$ y $72^{\circ} 30^{\prime} 34,98^{\prime \prime} \mathrm{W}$ ) realizando actividades educativas y búsqueda de triatominos durante noviembre 2008 y mayo 2009. Los especímenes se identificaron según la clave taxonómica de Lent y Wygodzinsky (1979) y la infección se determinó por la observación directa de las heces y por examen molecular, utilizando los iniciadores 121 y 122 específicos para una región de kADN de T. cruzi.

Resultados. Se caracterizaron 135 viviendas, en las cuales se evidenció una frecuencia de construcción de $64 \%$ en paredes de ladrillo, 40,4 $\%$ parcialmente rebocadas, $64 \%$ con techo de cinc y $50 \%$ con pisos de cemento; el $23 \%$ tenía anexos. 
Había presencia de perros (37\%) y gallinas (29 $\%)$, principalmente. Se detectaron en total $55 E$. mucronatus en 10 viviendas (7,4\%); los factores asociados fueron las paredes con grietas y la presencia de animales domésticos. Dos viviendas presentaron ninfas (II, III y IV), exuvias y huevos de E. mucronatus. Todos los exámenes directos resultaron negativos para formas flageladas, pero cuatro grupos analizados molecularmente fueron positivos para T. cruzi.

Conclusiones. Se establece $E$. mucronatus como una especie de invasión progresiva al domicilio y verdadero riesgo para el establecimiento de la infección humana por T. cruzi.

$$
\text { - }-
$$

\section{Factores climáticos y distribución geográfica de especies de triatominos (Hemiptera: Reduviidae) en el estado de Guerrero, México}

E. Rodríguez-Bataz ${ }^{1,5}$, S. A. Pineda-Rodríguez ${ }^{1}$, M. Torres-Armenta ${ }^{1}$, R. Rosario-Cruz ${ }^{2}$, B. Nogueda-Torres ${ }^{3}$, J. A. Martínez-lbarra ${ }^{4}$, J. L. Rosas-Acevedo ${ }^{5}$

1 Laboratorio de Investigación en Parasitología, Universidad Autónoma de Guerrero, México

2 Laboratorio de Artropodología, INIFAP, México

3 Laboratorio de Parasitología, Instituto Politécnico Nacional, México

4 Centro Universitario del Sur, Universidad de Guadalajara, México

5 Unidad de Desarrollo Regional, México

Introducción. De las 141 especies de triatominos, México cuenta con al menos 34, en su mayoría diferentes de aquéllas distribuidas en el resto del continente americano. Se llevó a cabo un estudio en 33 localidades, para determinar las especies de triatominos y su distribución geográfica en relación con los factores climáticos.

Materiales y métodos. La captura de triatominos se hizo dentro y fuera de la vivienda, por el método hora/hombre, y la identificación, con las claves de Lent y Wygodzinsky. Se tomaron lecturas de las variables temperatura, altitud, coordenadas geográficas y datos de la Comisión Nacional de la Biodiversidad.

Resultados. En 18 localidades se recolectaron triatominos de los géneros Triatoma ( $T$. barberi y $T$. dimidiata)y Meccus (M. pallidipennisy M. mazzottii). Meccus pallidipennis (58,9\%), fue la especie más abundante y más distribuida (42,4\%), seguida de M. mazzottii (33,3 \%), Triatoma barberi (18,2 \%) y $T$. dimidiata (3\%). Las especies recolectadas mostraron una distribución característica según la especie. Meccus pallidipennis y $T$. barberi se distribuyen en altitudes más elevadas, en rangos que oscilan entre los 400 y los 1.400 msnm. M. pallidipennis se encontró en un rango más amplio de altitud, al distribuirse entre los 400 y los 1.400 msnm, y T. barberi, entre los 600 y los 1.400 msnm. En altitudes bajas, se presentaron $M$. mazzottii (0 a 1.000 msnm) y $T$. dimidiata (400 a $600 \mathrm{msnm}$ ). En relación con el clima, se observó que $M$. pallidipennis se distribuye principalmente en clima templado húmedo, al igual que T. barberi, en tanto que M. mazzottii lo hace en clima cálido subhúmedo intermedio.

Conclusión. Las diferentes especies se distribuyen a altitudes distintas y bajo condiciones climáticas disímiles.

\section{Actualización de la distribución de especies de triatominos en el departamento del Atlántico, Colombia, 2003-2010}

Ronald Maestre-Serrano ${ }^{1}$, Melisa Eyes-Escalante ${ }^{2}$

1 Grupo de Investigación en Enfermedades Tropicales y Biomédicas del Atlántico, Secretaría de Salud del Atlántico, Barranquilla, Colombia

2 Grupo de investigación Biodiversidad del Caribe Colombiano, Universidad del Atlántico, Barranquilla, Colombia

Introducción. La enfermedad de Chagas, o tripanosomiasis americana, es una enfermedad transmitida por vectores de interés en salud pública para Colombia. El departamento del Atlántico es considerado no endémico para este evento; sin embargo, existen factores de riesgo, como la presencia de especies de triatominos.

El presente trabajo consistió en actualizar la distribución de especies de triatominos en áreas urbanas, periurbanas y rurales del departamento del Atlántico, Colombia, durante los años 2003 a 2010.

Metodología. Se hizo un estudio descriptivo y retrospectivo que consistió en analizar los datos de registros de triatominos para el departamento del Atlántico, entre los años 2003 al 2010, a partir de la información obtenida del sistema de vigilancia entomológica para la enfermedad de Chagas del Laboratorio de Salud Pública del Atlántico y los registros del grupo de investigación Biodiversidad del Caribe Colombiano, de la Universidad del Atlántico.

Todos los especímenes se determinaron taxonómicamente a partir de caracteres morfológicos y siguiendo las claves taxonómicas de Lent y Wygodzinsky (1979). 
Resultados. Durante el periodo estudiado se recolectaron 46 ejemplares de triatominos, correspondientes a las especies Panstrongylus geniculatus $(91 \%)$ y Eratyrus mucronatus $(7 \%)$. Los registros correspondieron a los municipios de Piojó, Tubará y Puerto Colombia, en la zona costera del departamento del Atlántico; Soledad, en la zona rivereña, límites con el departamento de Magdalena, y Luruaco, al sur del departamento en límites con el de Bolívar.

Conclusiones. Se actualizó la distribución de especies de triatominos en el departamento del Atlántico, con el hallazgo de las especies $P$. geniculatus y $E$. mucronatus en ambientes urbanos, periurbanos y rurales. 